\title{
RADICAL BEHAVIOR AND THE WEDDERBURN FAMILY
}

\author{
BY F. J. FLANIGAN \\ Communicated by Alex Rosenberg, June 7, 1972
}

1. A question. One sometimes constructs a family of algebras (e.g. various group algebras) and then hopes to prove that all its members have zero radical. If this is false, then one may attempt to describe the radicals that occur. Here we discuss the reverse process. Encouraged by the dictum 'When you have a lemon, make a lemonade,' we identify the following problem. Given a nonzero nilpotent algebra $N$, describe the set of unital algebras $A$ satisfying the equation

$$
\operatorname{rad} A=N
$$

(together with a certain nontriviality condition; see (2.1)). If the underlying scalar field $k$ is perfect, then the Wedderburn Principal Theorem implies that (1.1) is equivalent to the search for semisimple $S$ whose multiplication "associates" with that of $N$ so that the semidirect sum

$$
A=N+S
$$

is associative (and has no "useless" semisimple ideal summands; see (2.1)). Thus we are curious about nontrivial extensions of the trivial process of adjoining a unit to an algebra that lacks one.

Some basic intuitions about (1.2): (a) If $S$ is to be complicated, then the given $N$ should be relatively uncomplicated. For instance, if $S$ has orthogonal idempotents $e_{\alpha}, e_{\beta}$, then the subspace $e_{\alpha} \cdot N \cdot e_{\beta}$ must have zero square (very uncomplicated). (Is there a conservation law?) (b) If a more complicated ("generic") nilpotent algebra associates with the semisimple $S$, then every less complicated nilpotent specialization should do likewise. (c) The collection of maximal $S$ satisfying (1.2) is a reasonable structural invariant, yielding insight into the overall decomposability of $N$ as an algebra with operators.

The main results announced here: Theorem (2.6) relating solutions $S$ of (1.2) for a fixed $N$ to solutions for its graded form gr $N$; Theorems (3.1) and (4.1), which solve (1.2) in the cases of commutative indecomposable $N$ and square-zero $N$, respectively (see also (6.1) for maximal $S$ ); the deformation theorem (5.1) which makes precise our intuition (b) above; the stability result (7.2) relating idealhood in $N$ with that in $A=N+S$, and reducing the general problem (1.2) to the case of indecomposable $N$;

AMS (MOS) subject classifications (1970). Primary 16A21, 16A22; Secondary 16A58. 
the "uniqueness of maximal solutions" conjecture (6.3) for indecomposable noncommutative $N$; an upper bound (6.2) on the size of solutions $S$ in terms of the number of generators of $N$. We mention some further directions (fiber products, partial orderings) in the final section.

AdDED IN PRoof. Professor Marshall Hall, Jr. discussed algebras "bound to the radical" in Trans. Amer. Math. Soc. 48 (1940), 391-404.

2. Definitions and a basic lemma. Throughout $N \neq(0)$ is a finitedimensional nilpotent associative algebra of nilindex $v$ (minimal equation $N^{v+1}=(0)$ ) over the field $k$, and $S$ is a finite-dimensional semisimple $k$-algebra. We take $k$ algebraically closed, so that all solutions to (1.1) with $N=(0)$ (the homogeneous case) are known. Moreover, the $k$-space $N$ is an $S$-bimodule via a representation $\sigma$ of the enveloping algebra

$$
\sigma: S^{e}=S \otimes_{k} S^{\mathrm{op}} \rightarrow \operatorname{Lin}_{k} N .
$$

Thus we write $\sigma(a \otimes b) x=(a \cdot x) \cdot b=a \cdot(x \cdot b)$ for all $a, b$ in $S$ and $x$ in $N$.

(2.1) We say that $N$ accepts $(S, \sigma)$ as a nowhere trivial Wedderburn factor (briefly, $N$ accepts $S$ ) provided, for all $a, b$ in $S$ and $x, y$ in $N$, we have

Associativity. $a \cdot(x y)=(a \cdot x) y, x(a \cdot y)=(x \cdot a) y,(x y) \cdot b=x(y \cdot b)$;

Nowhere triviality. $a \cdot N=(0)$ implies $N \cdot a \neq(0)$, unless $a=0$.

Having this, we obtain a solution $A=N+S$ to (1.1) and (1.2). The nowhere triviality requirement prevents $N$ from accepting arbitrarily large $S$ (cf. (6.2)).

(2.2) If $N$ accepts both $(S, \sigma)$ and $(T, \tau)$, then these are strongly equivalent if there are $k$-algebra isomorphisms $f: S \rightarrow T$ and $\phi: N \rightarrow N$ such that

$$
(\phi \circ \sigma(a \otimes b)) x=\left(\left(\tau \circ f^{e}(a \otimes b)\right) \circ \phi\right) x
$$

for all $a, b$ in $S$ and $x$ in $N$. Strongly equivalent factors $(S, \sigma)$ and $(T, \tau)$ give rise to isomorphic algebras $A=N+S$ and $B=N+T$.

Note. The same $S$ may operate in inequivalent ways on $N$ and thereby give rise to nonisomorphic algebras. See $\S 4$.

(2.3) EXAMPLE. Let $N$ be the nilpotent algebra of all strictly upper triangular $n$ by $n$ matrices over $k$. Then $N$ accepts precisely those $(S, \sigma)$ which are strongly equivalent to a pair consisting of a subalgebra (same unity) of the algebra of all $n$ by $n$ diagonal matrices and its natural action on $N$. Note here that if $\operatorname{dim}_{k} N=1(n=2)$, then $N$ admits just two inequivalent Wedderburn factors, of dimensions 1 and 2.

(2.4) ExAMPLE. Let $N$ be the truncated polynomial ideal generated by $k$-independent possibly noncommuting elements $x_{1}, \ldots, x_{m}$ such that every monomial of degree $\geqq v+1$ reduces to zero. Then $N$ accepts $(S, \sigma)$ only if $S$ is the field $k \cdot 1$, with the single trivial exception $m=v=1$ noted in (2.3). 
(2.5) BASIC LeMmA. If $N$ accepts $(S, \sigma)$ as a nowhere trivial Wedderburn factor, then each quotient algebra $N / N^{i}$, with $2 \leqq i \leqq v+1$, accepts $\left(S, \sigma_{i}\right)$, where $\sigma_{i}$ is the usual induced representation of $S^{e}$.

Note that the subalgebras $N^{2}, \ldots, N^{v}$ need not accept $(S, \sigma)$ nowhere trivially, even if $N$ itself does. See (2.3).

The result about $N / N^{2}$ prompts our study $(\$ 4)$ of square-zero nilpotent algebras.

(2.6) THEOREM. If $N$ accepts $(S, \sigma)$ as a nowhere trivial Wedderburn factor, then its associated graded algebra $\operatorname{gr} N=\left(N / N^{2}\right) \oplus\left(N^{2} / N^{3}\right) \oplus$ $\cdots \oplus N^{v}$ accepts $\left(S, \sigma^{\prime}\right)$, where $\sigma^{\prime}$ is the representation induced from $\sigma$.

Nowhere triviality for $\left(S, \sigma^{\prime}\right)$ follows from (2.5) for $N / N^{2}$.

(2.7) Counterexample. The converse to (2.6) is false. For let $N$ be generated by commuting elements $x, y$ with minimal relations $x^{4}=0$, $y^{2}=x^{3}, x y=0$. Note $\operatorname{dim}_{k} N=4$. One checks that $N$ accepts only the 1-dimensional semisimple $S=k \cdot 1$. On the other hand, $\operatorname{gr} N \simeq$ $\left\langle\xi, \xi^{2}, \xi^{3}\right\rangle \oplus\langle\eta\rangle$ (gr $N$-direct), with $\xi^{4}=\eta^{2}=0$, and one readily sees that gr $N$ accepts 1-, 2- and 3-dimensional Wedderburn factors.

3. Commutative nilpotent algebras as radicals. The following result can be applied to the problem of computing the algebras with a given commutative radical.

(3.1) THEOREM. Let $k$ be algebraically closed, and suppose given a commutative nilpotent $k$-algebra $N$ which is $N$-indecomposable. Then either $N$ reduces to a line (and see (2.3)) or else $N^{2}>(0)$ and $N$ accepts the field $k \cdot 1$ but no other semisimple k-algebra, commutative or not.

4. The Wedderburn factors for the square-zero algebra. This case is important because of the necessity result (2.5) concerning the square-zero quotient algebra.

(4.1) TheOREM. Given $N \neq(0)$ with $N^{2}=(0)$. Let $S=S_{1} \oplus \cdots \oplus S_{s}$ where $S_{\alpha}$ is isomorphic to the simple algebra of all $r_{\alpha}$ by $r_{\alpha}$ matrices over $k$. Then $N$ accepts $(S, \sigma)$ as a nowhere trivial Wedderburn factor iff there exist nonnegative integers $X_{\alpha \beta}$ for $\alpha, \beta=1, \ldots, s$ satisfying

(i) $\sum_{\alpha} \sum_{\beta} r_{\alpha} X_{\alpha \beta} r_{\beta}=\operatorname{dim}_{k} N$,

(ii) $\sum_{\beta}\left(X_{\alpha \beta}+X_{\beta \alpha}\right)>0, \quad$ all $\alpha$.

This follows from a consideration of the Peirce decomposition of $N$ effected by the unity elements of the $S_{\alpha}$. In particular, $X_{\alpha \beta}=$ multiplicity of the irreducible $S_{\alpha} \otimes S_{\beta}^{o p}$ representation in $\sigma$.

Note that if $\operatorname{dim}_{k} N=r_{1} r_{2}$ then $N$ may serve as the "upper right-hand 
block" in the algebra of all $\left(r_{1}+r_{2}\right)$-square matrices with lower left-hand block zero (cf. $s=2$ ).

5. Generic deformations and their Wedderburn factors. The nilpotent generic member $N_{t}$ of a one-parameter family of deformations of $N$ is an algebra over the power series field $k((t))$ which specializes to $N$ when $t=0$. See Gerstenhaber's treatise (Ann. of Math. 79 (1964), 59-103) for details. Roughly speaking, the deformation $N_{t}$ will be less degenerate than $N$. For instance, if $N$ is a square-zero algebra and $B$ any $k$-algebra of the same dimension, then there is a deformation $N_{t}$ isomorphic over $k((t))$ to the scalar extension $B_{k(t))}=B((t))$. Thus the next result is intuitively appealing.

(5.1) THEOREM. If a generic deformation $N_{t}$ of $N$ accepts the semisimple $S((t))$ as a nowhere trivial Wedderburn factor over $k((t))$, then $N$ must accept $S$ over $k$.

One can re-obtain (2.6) using (5.1) and the fact that a filtered algebra is a (nongeneric) deformation of its associated graded algebra.

6. The question of maximal Wedderburn factors for $N$. Since semisimple subalgebras of accepted $(S, \sigma)$ are also accepted, it is the family of maximal Wedderburn factors which conveys essential information about $N$. We say $(S, \sigma)$ is maximal if every monomorphism $f: S \rightarrow T$ with $\sigma=\tau \circ f^{e}$ is an isomorphism. Here $(T, \tau)$ denotes a Wedderburn factor accepted by $N$.

(6.1) Theorem. Let $N^{2}=(0)$ and $S=S_{1} \oplus \cdots \oplus S_{s}$ as in (4.1). Then the following are equivalent:

(i) $(S, \sigma)$ is a maximal Wedderburn factor for $N$;

(ii) $s$ is even and, after re-indexing, $N=\bigoplus_{j=1}^{s / 2} S_{2 j-1} \cdot N \cdot S_{2 j}$, with each summand a simple $\left(S_{2 j-1}, S_{2 j}\right)$-bimodule;

(iii) $s$ is even and, after re-indexing, the multiplicity matrix $\left(X_{\alpha \beta}\right)$ of (4.1) reads $1,0,1,0,1, \ldots, 0,1$ down the principal superdiagonal with 0 's elsewhere.

(6.2) THEOREM. If $N$ (arbitrary) accepts $S$ as a nowhere trivial Wedderburn factor, then $\operatorname{dim}_{k} S \leqq \gamma^{2}+1$, where $\gamma=$ number of generators of $N=$ $\operatorname{dim}_{k}\left(N / N^{2}\right)$.

(6.3) Open question. If $N$ is indecomposable, is there a unique maximal nowhere trivial Wedderburn factor accepted by $N$ ? For evidence, see the commutative theorem (3.1), the strict upper triangular matrices (2.3), and (2.4). This question is important in the light of the reductions below.

7. Decomposability of $N$ and maximality of $S$. These next results allow us to concentrate on indecomposable nilpotent algebras. 
(7.1) Theorem. Let $N=\bigoplus P_{i}$ (ideal direct sum). If $T^{(i)}$ is a maximal Wedderburn factor for $P_{i}$, then $S=\bigoplus T^{(i)}$ is a maximal Wedderburn factor for $N$.

We say that $N$ is reduced if it has no proper square-zero $N$-ideal direct summands.

(7.2) Theorem (Stability of Summands). Let $N=\bigoplus P_{i}$ (ideal direct sum) accept $S$, thereby forming the unital associative algebra $A=N+S$. Then

(a) each $P_{i}^{2}$ is an $A$-ideal (although $P_{i}$ need not be);

(b) if $N$ is reduced, then also $N$ decomposes as $\oplus Q_{i}$ with $Q_{i} \simeq P_{i}$, $Q_{i}^{2}=P_{i}^{2}$, and each $Q_{i}$ an $A$-ideal;

(c) if $S$ is maximal for $N$ (reduced), then $S=\bigoplus T^{(i)}$ where $T^{(i)}$ is a maximal Wedderburn factor for $Q_{i}$.

Note that (b) and (c) are false for square-zero algebras of dimension $\geqq 2$, and also that (c) requires maximality.

8. Further observations and questions. Our chief unanswered question is (6.3). We also mention

(8.1) Which (indecomposable) $N$ admit only the trivial Wedderburn factor $S=k \cdot 1$ ? Cf. (3.1).

(8.2) Fiber products in the Wedderburn family. We have been able to define fiber products of Wedderburn factors $(S, \sigma)$ and $(T, \tau)$ over $\operatorname{Lin}_{k} N$ in certain cases. These would be of greater interest if the answer to the uniqueness question (6.3) is negative. In this event the partial ordering on the family of $(S, \sigma)$ must also be studied.

Department of Mathematics, University of California, San Diego, la Jolla, CALIFORNIA 92037 